\title{
Quelles piscicultures demain ?
}

\section{Christian Lévêque \\ Président de l'Académie d'agriculture (2013)}

DR émérite IRD

1 rue de Marnes

92410 Ville d'Avray

France

$<$ christianleveque2@orange.fr>
Pour répondre à la croissance démographique des prochaines décennies il faudra cultiver de plus grandes surfaces. Mais l'accroissement des surfaces cultivables, ou des pâturages, apparaît limité. On doit donc se poser sérieusement la question : quelle sera la contribution des produits d'origine aquatique à l'alimentation dans les prochaines décennies?

On sait que la pêche va mal, et que les stocks de poissons marins ou continentaux sont surexploités, à l'exemple de la morue de Terre-Neuve. On pêche de plus en plus loin et de plus en plus profond, sur des stocks réputés vulnérables. Dans ce contexte, quel peut être le rôle de l'aquaculture ? Le poisson d'élevage peut-il se substituer aux pêches marines qui s'épuisent (certains parlent même de les sauver). On sait que l'aquaculture connaît une progression rapide et que la production aquacole devrait atteindre, à courte échéance, près de la moitié de la production halieutique, avec des marges de progrès encore considérables. Mais on sait aussi que l'essentiel de la production aquacole se fait en eaux douces et saumâtres, ce qui signifie qu'elle ne se substitue pas aux poissons marins. Contrairement à certaines idées reçues, l'aquaculture ne permet donc pas de sauvegarder les espèces marines. Elle permet simplement, à l'heure actuelle, de fournir des protéines à une population humaine en forte croissance. C'est l'Asie qui fournit l'essentiel de cette production, avec essentiellement des cyprinidés, des siluriformes, et des tilapias.
Rappelons qu'il existe un paradoxe en matière de poissons: le nombre d'espèces de poissons d'eaux douces et saumâtres est à peu près équivalent au nombre d'espèces de poissons marins, alors que les eaux douces n'occupent que $0,01 \%$ de la surface du globe contre $70 \%$ pour le milieu marin.

Cette grande diversité des espèces d'eau douce peut en partie s'expliquer par la fragmentation des milieux continentaux dont l'isolement favorise la spéciation. Elle représente un atout pour l'aquaculture car on observe une grande diversité de comportements et d'adaptations écologiques et physiologiques qui peuvent être utiles pour la domestication. Il existe ainsi un grand potentiel en matière de recherche d'espèces d'intérêt aquacole en milieu continental, ainsi que des marges importantes d'amélioration des espèces actuellement domestiquées (génétiques, physiologiques, etc..). C'est dans ces domaines de recherche qu'il faudrait beaucoup plus investir à des niveaux comparables à ce qui se fait dans l'amélioration du maïs par exemple!

Dans les pays tropicaux, la pression de la pêche combinée aux changements climatiques et aux aménagements (barrages) a entraîné une forte diminution des captures. À l'exemple du delta central du Niger, cette zone réputée autrefois fort poissonneuse et dans laquelle les pêcheurs ne trouvent plus actuellement de quoi survivre. Les sécheresses répétées, la réduction des surfaces inondables du fait des barrages, limitent les capacités de reproduction des poissons. Sans accès 
facile au foncier, les pêcheurs sont condamnés à surexploiter les maigres stocks encore disponibles, ou à émigrer. Ajoutons que les autorités administratives, avant et après les indépendances, ont pendant longtemps privilégié une gestion centralisée des pêches, selon des modèles occidentaux très mal adaptés aux situations locales. En voulant imposer cette gestion étatique elles ont marginalisé ou tout simplement éliminé les modes de gestion traditionnels qui s'appuyaient sur des interdits et des tabous pour réguler les périodes et les lieux de pêche ainsi que les engins autorisés. Il en résulte une quasi-absence de régulation, et l'utilisation mal contrôlée d'engins prohibés, voire de produits toxiques.

Pourtant, l'espèce phare de la pisciculture continentale est actuellement le tilapia, originaire d'Afrique, dont on maitrise bien la reproduction et la croissance en captivité. Différentes souches font aussi l'objet d'amélioration génétique. Mais les tilapias sont produits massivement en Asie à l'heure actuelle, alors que leur production reste modeste en Afrique. Une question récurrente est de comprendre pourquoi la pisciculture a autant de mal à s'implanter en Afrique malgré de nombreux efforts en matière de transfert des technologies et de formation de pisciculteurs. D'abord axée sur le développement de l'aquaculture villageoise, la production de poissons s'oriente actuellement vers une production plus intensive, de type industriel, avec certains projets importants, notamment en Afrique de l'Est, qui semblent connaître quelques succès.
L'effort de recherche scientifique et technique sur les espèces de poissons d'eau douce susceptibles d'être utilisables en aquaculture continentale doit être poursuivi et intensifié. D'autant plus que, par rapport aux poissons marins actuellement domestiqués, beaucoup de poissons d'eau douce offrent un avantage comparatif important: ils ne nécessitent pas d'être alimentés par des protéines animales, mais utilisent surtout les déchets des industries agricoles. Car l'élevage des poissons marins carnivores (dorades, turbot, thon, loup, etc.), ceux qui sont les plus appréciés sur le marché, est tributaire de la pêche des petits poissons (les poissons fourrage) capturés en grandes quantités (37\% des captures mondiales). Une situation qui ne peut perdurer compte tenu des conséquences sur les écosystèmes marins. 